\title{
Consumo de alcohol, conducta antisocial e impulsividad en adolescentes españoles
}

\author{
Natalia Díaz García y María de la Villa Moral Jiménez ${ }^{*}$ \\ Universidad de Oviedo, España.
}

Recibido, julio 27/2016

Concepto de evaluación, septiembre 21/2017

Aceptado, octubre 2/2017
Referencia: Díaz García, N. \& Moral Jiménez, M.V. (2018). Consumo de alcohol y conducta antisocial e impulsividad en adolescentes Españoles. Acta colombiana de Psicología, 21(2), 110-120. doi: http:// www.dx.doi.org/10.14718/ACP.2018.21.2.6

\section{Resumen}

La relación entre el consumo de alcohol y la conducta antisocial en adolescentes ha sido ampliamente reportada en investigaciones anteriores. El objetivo principal de este estudio es evaluar dicha relación, además de estudiar cómo influye en ella la impulsividad. Se utilizó la Escala Rutgers Alcohol Problem Index (RAPI) para evaluar el consumo de alcohol, la Escala de Conducta Antisocial y Delictiva en Adolescentes, y la Escala de Impulsividad de Barratt para la conducta antisocial. Se hizo un muestreo no probabilístico de tipo intencional que resultó en una muestra compuesta por 212 adolescentes con edades entre los 12 y los 18 años $(\mathrm{M}=14.1, \mathrm{DE}=1.48)$. Los resultados mostraron que las variables que mejor predicen la conducta antisocial en adolescentes son el consumo de alcohol y la impulsividad cognitiva; y se comprobó que existen diferencias significativas en la conducta antisocial según el género, pero no en función del consumo de alcohol. Respecto al nivel de edad, se encontraron diferencias significativas entre todos los niveles de edad en el consumo de alcohol; así como diferencias en la conducta antisocial entre la adolescencia temprana y la adolescencia media, y entre la adolescencia temprana y la adolescencia tardía. Se discuten las posibles implicaciones de la vinculación entre el consumo de alcohol y el delito, en virtud de los resultados obtenidos.

Palabras clave: adolescencia, consumo de alcohol, conducta antisocial, impulsividad.

\section{Alcohol consumption, antisocial behavior and impulsivity in Spanish adolescents}

\begin{abstract}
The relationship between alcohol use and antisocial behaviour in teenagers has been widely reported in previous research. Our main aim was to evaluate this relationship, and also to study how impulsivity influences it. The instruments used were the Rutgers Alcohol Problem Index (RAPI), to evaluate alcohol consumption; the Scale of Antisocial and Criminal Behaviour in Teenagers, and the Barratt Impulsiveness Scale, to assess these behaviors in the youth. The sampling method was non probabilistic and intentional, and the sample consisted of 212 teenagers aged between 12 and 18 years (Average=14.1, $\mathrm{SD}=1.48$ ). The results showed that the variables which better predict anti-social behaviour in teenagers are alcohol consumption and cognitive impulsiveness. On the other hand, it was found that there are significant differences in antisocial behavior according to gender, but not in terms of alcohol consumption. Regarding age level, significant differences were found in alcohol consumption between all age levels, and in antisocial behavior are concentrated between early and medium adolescence, and between early and late adolescence. The possible implications of the link between alcohol consumption and crime by virtue of the results obtained are discussed.
\end{abstract}

Key words: adolescence, alcohol consumption, antisocial behaviour, impulsiveness.

\footnotetext{
* Facultad de Psicología Plaza Feijóo, s/n, despacho 211. Tel.: +34 985103282, mvilla@uniovi.es
} 


\title{
Consumo de álcool, conduta antissocial e impulsividade em adolescentes espanbóis
}

\begin{abstract}
Resumo
A relação entre o consumo de álcool e a conduta antissocial em adolescentes tem sido amplamente relatada em pesquisas anteriores. O objetivo principal deste estudo é avaliar tal relação, além de estudar como a impulsividade tem influência nela. Utilizou-se a Escala Rutgers Alcohol Problem Index (Rapi) para avaliar o consumo de álcool, a Escala de Condutas Antissociais e Delitivas em Adolescentes e a Escala de Impulsividade de Barratt para a conduta antissocial. Foi feita uma amostragem não probabilística de tipo intencional que resultou em uma amostra composta por 212 adolescentes com idades entre 12 e 18 anos $(M=14.1, \mathrm{DP}=1.48)$. Os resultados mostraram que as variáveis que melhor predizem a conduta antissocial em adolescentes são o consumo de álcool e a impulsividade cognitiva; ainda, ficou comprovado que existem diferenças significativas na conduta social de acordo com o gênero, mas não em função do consumo de álcool. Com relação ao nível de idade, foram encontradas diferenças significativas entre todos os níveis de idade no consumo de álcool, assim como diferenças na conduta antissocial entre a adolescência precoce e a adolescência média, e entre a adolescência precoce e a adolescência tardia. Discutem-se as possíveis implicações da associação entre o consumo de álcool e o delito em virtude dos resultados obtidos.

Palavras-chave: adolescência, consumo de álcool, conduta antissocial, impulsividade.
\end{abstract}

\section{INTRODUCCIÓN}

La adolescencia es un periodo vital en el que el hábito social de consumo de alcohol es mucho más probable (Andrade, Betancourt, Moreno \& Alvis, 2017; Gázquez et al., 2016; Londoño \& Valencia, 2012; Moral, Rodríguez $\&$ Sirvent, 2006). Este periodo de transición entre la infancia y la adultez está caracterizado por una "cultura de edad" de la que el adolescente se siente miembro y en la que va conformando sus propios comportamientos, interiorizando valores y compartiendo espacios y tendencias normativas, todo lo cual contribuye a la conformación de su identidad psicosocial. Durante esta etapa comienza la toma autónoma de decisiones en aspectos relativos a los vínculos interpersonales, la pertenencia a determinados grupos de iguales y la propia construcción de aspectos identitarios (Faílde, Dapía, Alonso \& Pazos, 2015; Pedreira, Blanco, Pérez-Chacón \& Quirós, 2014).

Con respecto a las prácticas de ocio y la experimentación con sustancias psicoactivas, en el informe de la Organización Mundial de la Salud (OMS, 2014) se señala que el consumo de alcohol representa una amenaza para la salud pública, y que son múltiples los factores asociados a su consumo, como las urgencias en lesionados de tráfico con alcoholemia positiva, intoxicaciones agudas, altercados violentos, suicidios, conductas infractoras y conductas sexuales de riesgo, entre otras (Bejarano \& Sáez, 2008; Farke \& Anderson, 2007; Galicia, Alonso \& Nogué, 2014; Isorna, Fariña, Sierra \& Vallejo-Medina, 2015).

Ciertamente, el consumo de alcohol en la adolescencia es considerado uno de los problemas de salud pública más graves, ya que en esta edad aumentan las posibilidades de que dicho consumo se mantenga o agudice durante la edad adulta, o que se pase al consumo de otras sustancias psicoactivas (Laespada, 2010; Musitu, Suárez, Del Moral, Martínez \& Villareal, 2015; Natera, Juárez, Medina, Mora \& Tiburcio, 2006). Al respecto, en el macroestudio con estudiantes colombianos de Scoppetta, Pérez y Lanziano (2011) se obtuvieron perfiles de consumo en función de cada etapa de la adolescencia, donde se constataron mayores riesgos en adolescentes que presentan un inicio de consumo más temprano y con mayores desajustes previos.

Por otra parte, en el entorno europeo es cada vez más habitual la modalidad de consumo concentrado, o Binge Drinking, que consiste en la ingesta de grandes cantidades de alcohol en pocas horas, mayoritariamente en momentos de ocio de fin de semana, y con el fin de mantener cierto nivel de embriaguez y cierto grado de pérdida de control (Cortés, Espejo, Martín \& Gómez-Íñiguez, 2010; Farke \& Anderson, 2007; Motos, Cortés, Giménez \& Cadaveira, 2015). En España, el Consumo Intensivo de Alcohol (CIA) en jóvenes se asocia con el politoxiconsumo y representa un factor de riesgo para el desarrollo posterior de abuso y dependencia alcohólica (Balodis, Potenza \& Olmstead, 2009). Y con respecto a las urgencias hospitalarias relacionadas con el alcohol en jóvenes, en España se detecta una clara presencia de las urgencias en los fines de semana o festivos (Sánchez, Redondo, García \& Velázquez, 2012); pues, aunque los adolescentes que consumen más alcohol disponen de información acerca de los efectos negativos del mismo, parece que presentan una percepción distorsionada de los efectos negativos derivados del consumo (Moral \& Ovejero, 2011; Morales et al., 2015).

En el último informe de la Organización Mundial de la Salud (2014) sobre consumo de alcohol se indica que se 
está produciendo un cambio importante en los patrones de consumo de las sociedades occidentales. En este sentido, en estudios recientes se reporta que el inicio de consumo es cada vez más temprano, que hay cambios en el patrón de consumo y que el consumo entre ambos géneros es más equitativo (ESTUDES, 2014; López \& Rodríguez-Arias, 2010; OEDT, 2015; Romo, Marcos, Gil, Marquina \& Tarragona, 2015).

Asimismo, tal como lo señalan Villarreal-González, Sánchez-Sosa y Musitu (2013), el consumo de alcohol en grupo es ya parte de la cultura juvenil, y esto implica una concepción específica del espacio y tiempo construido por ellos mismos a través de la interacción, que refleja ciertas normas y valores colectivos. Incluso, de acuerdo con los resultados de la Encuesta sobre Uso de Drogas en Estudiantes de Enseñanzas Secundarias (ESTUDES, 2014), se encontró que seis de cada diez estudiantes con edades entre los 14 y 18 años se han emborrachado alguna vez, y que tres de ellos lo hicieron en el último mes.

También, según datos de la Encuesta Domiciliaria sobre Alcohol y otras Drogas (EDADES) (Observatorio Español de la Droga y las Toxicomanías, 2015a), el alcohol está presente en el $95 \%$ de los policonsumos; $y$, se constata, sigue siendo la sustancia psicoactiva más consumida -incluso, registra un ligero incremento-. Asimismo, desde el Observatorio Español de la Droga y las Toxicomanías (2015b) se indica que el alcohol es la sustancia psicoactiva con el consumo más generalizado entre la población española; y que, en el 2015, el $93.1 \%$ de las personas de 15 a 64 años había tomado bebidas alcohólicas en alguna ocasión, porcentaje que se ha ampliado ligeramente respecto al 2011, por lo que la edad media de inicio de consumo se sitúa en los 16.7 años; edad similar a la obtenida en años anteriores. Todo ello supone una auténtica emergencia sociosanitaria (Moral, Bringas, Ovejero, Morales \& Rodríguez, 2017).

Con respecto al género, parece que hay una reducción de la brecha en los consumos intensivos por parte de las mujeres (OMS, 2014), siendo esto aún más evidente en la adolescencia. Específicamente, en la Encuesta Estatal sobre el Uso de Drogas en Estudiantes de Enseñanza Secundaria(ESTUDES, 2014/2015) se señala que el $1.7 \%$ de los adolescentes de 14 a 18 años ha consumido alcohol diariamente en el último mes; y que hay una creciente incorporación de las chicas al consumo de drogas legales, quienes incluso sobrepasan a los chicos en consumo de alcohol, tranquilizantes y tabaco, puesto que el porcentaje de chicas de entre 14 y 16 años que se emborracha es mayor que el de los varones de la misma edad. De esto se puede concluir que, de acuerdo con Romo et al. (2015), aunque en un principio los excesos en consumo de alcohol fueron asociados al género masculino, en la actualidad ya no se considera algo exclusivo y las mujeres ejercen un papel más significativo.
Ahora bien, en esta etapa vital, otro de los comportamientos de riesgo más estudiado ha sido la conducta juvenil delictiva (Bringas, Rodríguez, Moral, Sánchez \& Ovejero, 2012; Muñoz et al., 2011; Rodríguez, Rodríguez-Franco, López-Cepero \& Bringas, 2012; Rodríguez, Ovejero, Bringas \& Moral, 2016), comportamiento asociado al consumo de sustancias psicoactivas en adolescentes, tema que ha originado creciente interés en los últimos años (Delisi, Angton, Behnken \& Kusow, 2015).

Al respecto, distintas investigaciones han encontrado un consistente patrón de consumo de variadas sustancias en menores con conductas antisociales (Contreras, Molina \& Cano, 2012; Ramírez, 2003; Ribas et al., 2015; San Juan, Ocáriz \& Germán, 2009); y, de acuerdo con Loeber (1988), un importante predictor del abuso de sustancias puede ser la aparición de la conducta antisocial en edades tempranas -que continuará con el inicio de la adolescencia-; sin embargo, en otras investigaciones los resultados indican una relación inversa, es decir, que sería la edad de inicio de consumo la variable predictora de futuros actos violentos -así, se dice que los adolescentes con conducta violenta comenzaron a consumir sustancias psicoactivas en edades más tempranas(Rodríguez, Bringas, Moral, Pérez \& Estrada, 2012).

Independientemente de lo que haya sido primero, lo cierto es que la violencia juvenil en contextos de ocio es preocupante, y es un hecho cada vez más frecuente (Bellis, Hughes, Korf \& Tossman, 2004; Blay et al., 2010). Al respecto, distintos autores plantean que el incremento de la violencia se asocia con la expansión del modelo de diversión relacionado con el consumo de alcohol y drogas (Anderson, Hughes \& Bellis, 2007; Blay et al., 2010), y que los contextos recreativos nocturnos se relacionan con el consumo abusivo de drogas y con otros riesgos para la salud, como la violencia (Calafat, Fernández, Juan \& Becoña, 2007; Farke \& Anderson, 2007).

Teniendo esto en cuenta, el análisis de las conductas de riesgo en adolescentes ha sido asociado a mecanismos sociales, emocionales y cognitivos del desarrollo humano en estudios recientes (Dussaillant, 2010). Y, partiendo de acá, podría decirse que los esquemas cognitivos, especialmente cuando se asocian a un estilo impulsivo de resolución de problemas, podrían considerarse como un factor de riesgo para el consumo de sustancias y ciertas conductas antisociales (Calvete \& Estévez, 2009).

Al respecto, según Velázquez, Arellanez y Martínez(2012), estos esquemas funcionan facilitando contenidos cognitivos relacionados con el deseo de obtener recompensas inmediatas, lo cual, unido a un estilo impulsivo, haría que no se diera la reflexión adecuada acerca de las consecuencias de las conductas de riesgo. Razón por la cual se sugiere la relación entre 
el uso de drogas, una baja asertividad, un menor control de impulsos y desajustes en el manejo de las emociones.

Ciertamente, la impulsividad se ha relacionado con numerosos trastornos, como el abuso de sustancias (MartínezLoredo, Fernández-Hermida, Fernández-Artamendi, Carballo \& García-Rodríguez, 2015), tema que ha tomado gran importancia, ya que en los estudios anteriores se ha comprobado una asociación entre la presencia del uso de sustancias y otras conductas de riesgo durante la adolescencia (Peters et al., 2015). Adicionalmente, se ha evidenciado una relación entre el bajo autocontrol y la conducta delictiva, así como con la permanencia de semejante conducta infractora (Contreras, Molina \& Cano, 2011; Mulvey, Shubert \& Chassin, 2010); así como el hecho de que adolescentes con conducta antisocial o delictiva muestran alta impulsividad y bajo autocontrol (Sanabria \& Uribe, 2009); y que el perfil de consumo de alcohol y la alta impulsividad representan una patología dual común en jóvenes que han cometido delitos, por lo que podrían considerarse como jóvenes multiproblemáticos (Ribas et al., 2015).

Teniendo lo anterior en cuenta, en la presente investigación se planteó como objetivo general analizar las variables que pueden predecir la conducta antisocial en población adolescente; y, como objetivos secundarios, valorar la importancia relativa de cada una de esas variables en la determinación del fenómeno, estimar el porcentaje de las diferencias en conducta antisocial que se podría predecir con las mismas, y comprobar si existen diferencias significativas en cuanto al género y la edad en la conducta antisocial y el consumo de alcohol.

Siguiendo con esto, se parte de la hipótesis de que las variables que mejor predicen la conducta antisocial en adolescentes son el consumo de alcohol y la impulsividad; que hay mayores puntuaciones en consumo de alcohol por parte de las mujeres, así como mayores puntuaciones en conducta antisocial por parte de los varones; y que se presentan mayores puntuaciones, de manera significativa, en el grupo de edad de la adolescencia tardía con respecto al de adolescencia temprana y media en las variables de consumo de alcohol y conducta antisocial.

\section{MÉTODO}

\section{Participantes}

La muestra estuvo compuesta por 212 adolescentes de Enseñanza Secundaria de la ciudad de Oviedo (Principado de Asturias, España) que contestaron el cuestionario de manera anónima con sus respectivos datos sociodemográficos, necesarios para el análisis de los datos. Para la selección de la muestra se siguió una serie de criterios de inclusión, accesibilidad y consentimiento, con un muestreo bietápico de conveniencia con submuestreo aleatorio intragrupo. De los participantes, el $50.94 \%$ eran hombres $(n=108)$ y el $49.06 \%$ restante fueron mujeres $(\mathrm{n}=104)$. El rango de edad se situó entre los 12 y 18 años, siendo la media de 14.1 años $(\mathrm{DT}=1.48)$. $\mathrm{Y}$, con respecto al nivel de estudios cursados por los participantes, el $87.74 \%$ cursaban estudios de Educación Secundaria (de los 12 a los 16 años) y el $12.26 \%$ restante eran estudiantes de Bachillerato (cursos adicionales a la Educación Secundaria).

\section{Instrumentos}

Con el fin de alcanzar los objetivos planteados se hizo uso de la escala validada Rutgers Alcohol Problem Index (RAPI), de White y Labouvie (1989), la Escala de Conducta Antisocial y Delictiva en Adolescentes (ECADA), de Andreu y Peña (2013) y, la Escala de Impulsividad de Barratt (Barratt Impulsiveness Scale, BIS-11), de Barratt (2000).

Rutgers Alcohol Problem Index (RAPI) (White \& Labouvie, 1989). Esta prueba se utilizó para evaluar el consumo de alcohol en los adolescentes. Específicamente, se utilizó la validación al español realizada por López-Nuñez, Fernández-Artamendi, Fernández-Hermida, Campillo y Secades-Villa (2012), una herramienta de detección autoadministrada para estimar los problemas con el alcohol en los adolescentes que consta de veintitrés ítems en escala Likert con cuatro niveles de respuesta ( 0 es "nunca" o "casi nunca", 1 es "una o dos veces", 2 es "entre tres y cinco ocasiones" y 3 es "en cinco o más ocasiones"). En esta, los adolescentes deben indicar con qué frecuencia han experimentado, durante el año pasado, las situaciones allí descritas mientras consumían alcohol o como consecuencia de su consumo. Las propiedades psicométricas del instrumento en su conjunto mostraron un alfa de Cronbach de .87 (López-Nuñez et al., 2012); y, para la muestra de este trabajo, una consistencia interna alta, con un alfa de Cronbach de .91 .

Escala de Conducta Antisocial y Delictiva en Adolescentes (ECADA) (Andreu \& Peña, 2013). Este instrumento se utilizó con el fin de medir la conducta antisocial en adolescentes. La consistencia interna de la escala, obtenida a través del coeficiente alfa de Cronbach, fue de .86. Específicamente, las propiedades psicométricas del instrumento mostraron alta consistencia en cinco factores: Comportamiento predelictivo $(\alpha=.46)$, Comportamientos vandálicos $(\alpha=.67)$, Infracciones contra la propiedad $(\alpha=.66)$, Comportamiento violento $(\alpha=.58)$ y Consumo 
de alcohol y drogas $(\alpha=.61)$ (Andreu \& Peña, 2013). Para la muestra de este trabajo se obtuvo una consistencia interna, calculada con el alfa de Cronbach, de .82; y para los cinco factores se obtuvo una consistencia interna específica de .73 para Comportamiento predelictivo (Factor I), .71 para Comportamientos vandálicos (Factor II), .72 para Infracciones contra la propiedad (Factor III), .71 para Comportamiento violento (Factor IV) y .73 para Consumo de alcohol y drogas (Factor V).

Esta escala está integrada por 25 ítems con escala de respuesta de valoración dicotómica (verdadero/falso), donde el adolescente debe indicar la presencia o no durante el último año de las conductas descritas en los ítems de la escala, los cuales se encuentran agrupados en cinco dimensiones o factores, descritos por Andreu y Peña (2013) de la siguiente manera:

1. Comportamientos predelictivos (Factor I). Explora una serie de conductas no expresamente delictivas, aunque sí desviadas de las normas y reglas sociales (como faltar al colegio, fugarse de casa, conducir vehículos sin permiso o autorización, etc.).

2. Comportamientos vandálicos (Factor II). Incluye conductas claramente delictivas realizadas sobre objetos o propiedades (como daños en paradas de autobuses, mobiliario urbano, etc.).

3. Infracciones contra la propiedad (Factor III). Estima conductas delictivas como robos y hurtos en diferentes contextos y lugares (como la entrada sin permiso en una casa, edificio o propiedad privada).

4. Comportamiento violento (Factor IV). Registra conductas delictivas que implican la participación en agresiones contra personas y la posesión o uso de armas (como llevar una navaja, por ejemplo).

5. Consumo de drogas (Factor V). Estima el consumo de drogas vinculado a la conducta antisocial y delictiva (como consumir cannabis, cocaína o anfetaminas).

Escalade Impulsividadde Barratt(BIS-11) (Barratt, 2000).

Se utilizó la validación al español de esta prueba, realizada por Oquendo et al. (2001). Es un instrumento autoaplicado con el objetivo de evaluar la impulsividad; y consta de treinta ítems agrupados en tres subescalas de personalidad: Impulsividad cognitiva, Impulsividad motora e Impulsividad no planeada, medidos en una escala tipo Likert con cuatro opciones de respuesta (0 es "raramente o nunca", 1 es "ocasionalmente", 3 es "a menudo" y 4 es "siempre o casi siempre"), sin un punto de corte propuesto.

El análisis de la consistencia interna de la escala BIS11 fue realizado mediante el coeficiente alfa de Cronbach. En detalle, se obtuvo un valor de .78 para la totalidad del cuestionario, .60 para la subescala de Impulsividad cognitiva, .66 para Impulsividad motora, y .69 para Impulsividad no planificada (Oquendo et al., 2001). Mientras que para la muestra del presente trabajo se obtuvo una consistencia interna de .77 para la prueba completa, .77 para Impulsividad cognitiva,.78 para Impulsividad motora y, por último, .77 para Impulsividad no planeada.

\section{Procedimiento}

La recogida de datos se hizo a través del contacto previo de los investigadores con los equipos directivos y orientadores de los centros de Educación Secundaria, quienes llevaron a cabo la selección de las aulas participantes de manera totalmente aleatoria. Una vez seleccionadas las aulas, el tutor de cada curso autorizó la participación de los adolescentes y junto con la orientadora del centro elaboraron el calendario con las horas disponibles para la aplicación de los instrumentos. Finalmente, el cuestionario fue aplicado en la hora de tutoría semanal que tiene cada curso, en ausencia del profesor tutor para evitar que los alumnos se vieran influidos por su presencia a la hora de contestar el cuestionario. Las fechas de aplicación fueron del 8 al 21 de abril de 2016, día en el que se aplicó el cuestionario al último curso.

La versión final del cuestionario contenía una explicación al inicio en la que se decía que el cuestionario que iban a responder era de carácter totalmente anónimo y confidencial, así como el tiempo aproximado que les llevaría realizarlo, y se les pedía sinceridad en las contestaciones y la cumplimentación de todos los ítems. La participación de los adolescentes encuestados fue en todo momento voluntaria, contando con su consentimiento informado y respetando su anonimato. El estudio mantuvo a lo largo de su desarrollo una tasa de respuesta del $100 \%$.

\section{Análisis de datos}

Esta investigación presenta un diseño analítico, experimental y transversal, ya que la finalidad es determinar las variables explicativas de la relación entre el consumo de alcohol y la conducta antisocial en población adolescente mediante la contrastación de las hipótesis anteriormente descritas. Inicialmente, se comprobaron los supuestos paramétricos de normalidad y homocedasticidad con el fin de seleccionar las técnicas estadísticas adecuadas; así, tras emplear la prueba estadística Kolmogorov-Smirnov $(N=212)$, los resultados mostraron que los datos de las variables se ajustaban a una distribución normal. De ahí la necesidad de aplicar pruebas paramétricas.

Posteriormente, se realizó el análisis estadístico descriptivo (frecuencias, medias y desviación estándar), 
y con el fin de evaluar la consistencia interna de los instrumentos se escogió el alfa de Cronbach. Después, las comparaciones entre grupos se llevaron a cabo mediante varios tipos de técnicas: la prueba de regresión lineal múltiple, para comprobar qué variables predicen mejor la conducta antisocial en adolescentes, cuál es la importancia relativa de cada una de esas variables y qué porcentaje de las diferencias en conducta antisocial se podría predecir con las mismas; la T de Student, para comprobar si existían diferencias en la conducta antisocial y en el consumo de alcohol en función del género; y la prueba ANOVA de un factor, con el fin de determinar las diferencias según la edad en consumo de alcohol y conducta antisocial.

Por último, se calculó el tamaño del efecto con el estadístico d de Cohen en las pruebas de comparaciones de medias, así como el estadístico V de Cramer y el coeficiente de contingencia en el análisis de regresión para valorar la magnitud estimada de las relaciones propuestas. Para el procesamiento de los datos y su posterior análisis estadístico se utilizó el programa SPSS, versión 19.

\section{RESULTADOS}

En este apartado se recogen los resultados encontrados al contrastar las hipótesis planteadas. En primer lugar, se valora la importancia relativa de las variables investigadas en la determinación de la conducta antisocial en adolescentes, habiéndose predicho la importancia del consumo de alcohol y la impulsividad como variables explicativas. Y, en segundo lugar, se aportan los resultados relativos al análisis de las diferencias inter-género en consumo de alcohol y en conducta antisocial, así como en función del grupo de edad.
Primeramente, se comprobó que las variables predictoras Impulsividad cognitiva, Impulsividad motora, Impulsividad no planeada y Consumo de alcohol, al ser individualmente consideradas, presentan correlaciones significativas con el criterio "conducta antisocial" $(\mathrm{p}<.01)$. Por otra parte, con las cuatro variables es posible explicar un $36.6 \%$ de las diferencias en el criterio "puntuaciones en conducta antisocial"; donde el coeficiente de determinación múltiple es estadísticamente significativo $(\mathrm{p}<0,01)$.

Por otra parte, las variables Impulsividad motora e Impulsividad no planeada, aunque consideradas individualmente presentan una correlación significativa con el criterio, dejan de tenerla al entrar en la ecuación de regresión. Así, las principales variables explicativas en la ecuación de regresión son el Consumo de alcohol y la Impulsividad cognitiva. Además, con el consumo de alcohol es posible explicar un $30.1 \%$ de las diferencias en el criterio; y con las dos variables juntas se puede aumentar ese porcentaje hasta un $35.5 \%$. Con respecto al valor absoluto de los coeficientes beta, se encontró que para predecir la conducta antisocial es más importante el consumo de alcohol que la impulsividad cognitiva (véase Tabla 1).

Los resultados obtenidos al comparar los patrones de consumo de alcohol según el género (véase Tabla 2) mostraron que no existen diferencias intergénero significativas $(\mathrm{p}>.05)$, con un tamaño del efecto medio $(d$ de Cohen $=.36)$. Sin embargo, sí se comprueba la existencia de diferencias significativas en conducta antisocial en función del género, con un tamaño del efecto pequeño $(d$ de Cohen $=.25)$.

Por otra parte, para comprobar si el nivel de edad influye en el consumo de alcohol y en la conducta antisocial, lo primero que se llevó a cabo fue la recodificación de la variable edad en tres grupos o niveles de edad nuevos: adolescencia

Tabla 1

Variables explicativas de la conducta antisocial en adolescentes (análisis de regresión múltiple). Coeficientes en la ecuación final

\begin{tabular}{|c|c|c|c|c|c|}
\hline \multirow[b]{2}{*}{ Modelo } & \multicolumn{3}{|c|}{ Coeficientes no estandarizados } & \multicolumn{2}{|c|}{ Coeficientes tipificados } \\
\hline & $\mathrm{B}$ & Error típico & Beta & $\mathrm{t}$ & Sig. \\
\hline 1 & & & & & \\
\hline (Constante) & 1.899 & .010 & & 193.066 & .000 \\
\hline Consumo de alcohol & -.284 & .030 & -.549 & -9.511 & .000 \\
\hline 2 & & & & & \\
\hline (Constante) & 2.007 & .028 & & 72.420 & .000 \\
\hline Consumo de alcohol & -.246 & .030 & -.475 & -8.145 & .000 \\
\hline Impulsividad cognitiva & -.058 & .014 & -.243 & -4.160 & .000 \\
\hline
\end{tabular}

Nota. Variable dependiente: conducta antisocial. 
Tabla 2

Diferencias de medias según el género en el consumo de alcohol y la conducta antisocial (prueba T de Student)

\begin{tabular}{ccccccc}
\hline & Sexo & $\mathrm{N}$ & Media & DT & T de Student & $p$ \\
\hline \multirow{2}{*}{ Alcohol } & Hombre & 108 & .1546 & .28135 & -.159 & .873 \\
& Mujer & 104 & .1610 & .29975 & & \\
Conducta & Hombre & 108 & 1.8319 & .15610 & \multirow{2}{*}{-2.226} & .027 \\
antisocial & Mujer & 104 & 1.8773 & .14049 & & \\
\hline
\end{tabular}

Nota. $* \mathrm{p}<.05$.

temprana (12-13 años, $\mathrm{n}=81)$, adolescencia media $(14-15$ años, $n=89)$ y adolescencia tardía (16-18 años, $n=42$ ); $\mathrm{y}$, después, se realizó un ANOVA de un factor. Así, respecto a las diferencias en consumo de alcohol según la edad, se puede comprobar que la media más alta corresponde al grupo de la adolescencia tardía. Por lo que se confirma la existencia de diferencias significativas entre al menos dos niveles de edad comparados $\left(\mathrm{F}_{2,209}=26.082, \mathrm{p}<.01\right)$, $\mathrm{h}$ donde el tamaño del efecto es medio (eta cuadrado parcial $=.20$ ).

Adicionalmente, con el modelo desarrollado -que solo incluye el efecto del nivel de edad- es posible explicar el $20 \%$ de las diferencias en consumo de alcohol. Después, se consideraron los resultados de la prueba de contraste Games-Howell para comprobar entre qué grupos de edad se dan estas diferencias, puesto que no se cumple la homocedasticidad. Estos resultados están recogidos en la Tabla 3, donde se puede observar que hay diferencias significativas entre todos los niveles de edad, diferencias a favor de la adolescencia tardía con respecto al resto.
Por otra parte, en los resultados obtenidos en conducta antisocial según el nivel de edad se puede observar que la media más alta corresponde al nivel de edad asignado a la adolescencia temprana; $y$, por tanto, se constata la existencia de diferencias significativas entre al menos dos niveles de edad comparados $\left(\mathrm{F}_{2,209}=19.339, \mathrm{p}<.01\right)$, con un tamaño del efecto medio (eta cuadrado parcial $=.156$ ). Con el modelo desarrollado es posible explicar el 15.6\% de las diferencias en conducta antisocial debido al efecto del nivel de edad.

Finalmente, fueron considerados los resultados de la prueba de Scheffe para determinar entre qué grupos se dan las diferencias, ya que se cumplía el supuesto de homocedasticidad; y con esto se comprueba la existencia de diferencias significativas entre la adolescencia temprana y la adolescencia media $(\mathrm{p}<.01)(d$ de Cohen $=.64)$, y entre la adolescencia temprana y la adolescencia tardía $(\mathrm{p}<.01)$ $(d$ de Cohen $=1.01)$. Sin embargo, no se encontraron diferencias significativas entre la adolescencia media y la adolescencia tardía en 1 conducta antisocial (véase Tabla 4).

Tabla 3

Diferencias de medias en el consumo de alcohol según nivel de edad

\begin{tabular}{clll}
\hline Nivel de edad & \multicolumn{1}{c}{ Media } & DT & $\mathrm{n}$ \\
\hline Adolescencia temprana & .0317 & .10783 & 81 \\
Adolescencia media & .1632 & .30479 & 89 \\
Adolescencia tardía & .3892 & .35204 & 42 \\
Total & .1577 & .28985 & 212 \\
\hline (I) Nivel de edad & $(\mathrm{J})$ Nivel de edad & Sig. \\
\hline \multirow{2}{*}{ Adolescencia temprana } & Adolescencia media & $.001^{*}$ \\
Adolescencia media & Adolescencia tardía & $.000^{*}$ \\
& Adolescencia temprana & $.001^{*}$ \\
Adolescencia tardía & Adolescencia tardía & $.002^{*}$ \\
& Adolescencia temprana & $.000^{*}$ \\
\hline
\end{tabular}

Nota. $* \mathrm{p}<.05$ 
Tabla 4

Diferencias de medias en conducta antisocial según nivel de edad

\begin{tabular}{cccc}
\hline Nivel de edad & Media & DT & $\mathrm{n}$ \\
\hline Adolescencia temprana & 1.9249 & .12384 & 81 \\
Adolescencia media & 1.8279 & .14465 & .15139 \\
Adolescencia tardía & 1.7733 & .15003 & 42 \\
Total & 1.8542 & $(\mathrm{~J})$ Nivel de edad & 212 \\
\hline (I) Nivel de edad & Adolescencia media & $.000^{*}$ \\
Adolescencia temprana & Adolescencia tardía & $.000^{*}$ \\
& Adolescencia temprana & $.000^{*}$ \\
Adolescencia media & Adolescencia tardía & .112 \\
& Adolescencia temprana & $.000^{*}$ \\
Adolescencia tardía & Adolescencia media & .112 \\
\hline
\end{tabular}

Nota. $* \mathrm{p}<.05$

\section{DISCUSIÓN}

El primero de los objetivos del presente trabajo estaba centrado en comprobar qué variables predicen mejor la conducta antisocial en adolescentes. Con respecto a esto, se pudo comprobar que las variables que mejor predicen la conducta antisocial en adolescentes son el consumo de alcohol y la impulsividad cognitiva. Conclusión constatada en otras investigaciones que sugieren que la mayor capacidad predictiva del consumo de alcohol la tiene la impulsividad cognitiva (Contreras et al., 2012; San Juan et al., 2009).

Otro de los objetivos de esta investigación fue comprobar si existen diferencias significativas en cuanto al género y la edad en la conducta antisocial y en el consumo de alcohol. En virtud de los resultados encontrados, al comparar los patrones de consumo de alcohol según el género se pudo comprobar que no existen patrones inter-género diferenciales, y esto se encuentra en consonancia con otras investigaciones donde se habla de la incorporación creciente de las mujeres al consumo de alcohol, incluso superando a los varones (Romo et al., 2015); aspecto que, según la interpretación de Lyons y Willott (2008), puede deberse a que las mujeres consideran estos actos como un cambio en su posición en la sociedad actual.

Por otra parte, en cuanto a la conducta antisocial, se comprobó que sí existen diferencias significativas entre hombres y mujeres, siendo estas a favor de las mujeres. Al respecto, en investigaciones previas se han extraído resultados diversos, de modo que algunos estudios apuntan que el sexo masculino presenta más comportamientos antisociales y delictivos con una frecuencia y gravedad superior que las mujeres (López \& Rodríguez-Arias, 2010); mientras que otros, como Pozo (2012), constatan un acercamiento en las diferencias halladas, donde las chicas participan cada vez más en situaciones de violencia.

Con respecto a las diferencias según la edad de los adolescentes que han participado en el estudio, dado el interés de la investigación emplearon tres niveles (adolescencia temprana, adolescencia media y adolescencia tardía) y se pudo comprobar que en el consumo de alcohol existen diferencias significativas entre todos los niveles de edad, siendo estas diferencias a favor de la adolescencia tardía respecto al resto. En este sentido, diversas investigaciones previas coinciden en la importancia del papel de la edad, ya que señalan diferencias entre los adolescentes jóvenes y los adolescentes con más edad en los consumos (Cava, Murgui \& Musitu, 2008; García et al., 2012; López \& Rodríguez-Arias, 2010; Moral \& Ovejero, 2009); e, incluso, se ha señalado que los preadolescentes y los adolescentes tempranos no suelen incluirse en las investigaciones, a pesar de la gran importancia que tiene la identificación de los patrones de consumo y actuación entre los más jóvenes (Luengo et al., 2008).

Por otra parte, en la conducta antisocial también se encontraron diferencias significativas entre la adolescencia temprana y la adolescencia media, y entre la adolescencia temprana y la adolescencia tardía; sin embargo, la literatura científica sobre este tema parece no encontrar una explicación común, ya que no existe un acuerdo claro entre los autores en lo que se refiere a la edad de aparición y manifestación de la conducta antisocial. Por ejemplo, autores como Rechea (2008) señalan su inicio a partir de los 13 años, cuando incide en el carácter más o menos estable de las conductas antisociales hasta llegar a la adultez (Estévez et al., 2007), momento en el que se intensifican e, incluso, se desarrollan comportamientos delictivos (Cifuentes \& Londoño, 2011). 
Cabe señalar, adicionalmente, que el presente estudio presenta algunas limitaciones, como la selección de la muestra, que no es aleatoria y por tanto los resultados obtenidos no podrían ser extrapolados a toda la población adolescente, y que ha de circunscribirse a condiciones socioculturales donde resulten características las modalidades de consumo intensivo de alcohol. Además de esto, el estudio es de tipo transversal, lo que implica la imposibilidad de establecer causalidad; y otra de las limitaciones es que el cuestionario fue aplicado a los adolescentes en las respectivas aulas de los centros educativos, y esto puede suponer cierto sesgo de deseabilidad social.

Para finalizar, como propuesta investigadora se recomienda la conveniencia de incluir otras variables de análisis que constituyan factores de riesgo psicosocial tanto para el consumo intensivo de alcohol como para la conducta antisocial, centradas en aspectos identitarios y en factores de socialización familiar. Al respecto, siendo una problemática multideterminada, son numerosas las implicaciones derivadas de ambos objetos de estudio -consumo juvenil de alcohol y conducta antisocial en menores- tal como se ha constatado en diversas investigaciones (Moral \& Ovejero, 2009, 2011; Moral et al., 2017; Morales et al., 2015; Rodríguez et al., 2016), de ahí que resulte de sumo interés profundizar en el estudio comprehensivo e integral de la interrelación de ambas problemáticas con el fin de programar e implementar medidas de prevención e intervención psicosocial.

\section{REFERENCIAS}

Anderson, Z., Hughes, K., \& Bellis, M. A. (2007). Exploration of young people's experience y perceptions of violence in Liverpool's nightlife. Liverpool: Centre for Public Health, Liverpool John Moores University.

Andrade, P., Betancourt, D., Moreno, N. D., \& Alvis, A. (2017). Fortalezas externas y consumo de sustancias en adolescentes mexicanos y colombianos. Avances en Psicología Latinoamericana, 35(3), 515-529. DOI: http:// dx.doi.org/10.12804/revistas.urosario.edu.co/apl/a.4095

Andreu, J., \& Peña, M. (2013). Propiedades psicométricas de la Escala de conducta antisocial y delictiva en adolescentes: Desarrollo y validación. Anales de Psicología, 29, 516-522. DOI: http://dx.doi.org/10.6018/analesps.29.2.135951

Balodis, I. M., Potenza, M. N., \& Olmstead, M. C. (2009). Binge drinking in undergraduates: relationships with sex, drinking behaviours, impulsivity, and the perceived effects of alcohol. Behavioral Pharmacology, 20, 518-526. DOI: http://dx.doi.org/10.1097/FBP.0b013e328330c779

Barratt, E. S. (2000). Barratt Impulsiveness Scale, Version 11 (BIS 11). En Handbook of Psychiatric Measures (pp. 691693). Washington, DC: American Psychiatric Association.
Bejarano, J., \& Sáenz, M. (2008). Consumo problemático de alcohol en Costa Rica y su relación con antecedentes de abuso sexual. Acta Colombiana de Psicología, 11(1), 89-95.

Bellis, M. A., Hughes, K., Korf, D., \& Tossman, P. (2004). Violence in general places of entertainment. En Council of Europe (ed.): Violence and insecurity related to the consumption of psychoactive substances. Strasbourg: Pompidou Group, Council of Europe.

Blay, N., Calafat, A., Juan, M., Becoña, E., Mantecón, A., Ros, M., \& Far, A. (2010). Violencia en contextos recreativos nocturnos: su relación con el consumo de alcohol y drogas entre jóvenes españoles. Psicothema, 22(3), 396-402.

Bringas, C., Rodríguez, F. J., Moral, M. V., Sánchez, B., \& Ovejero, A. (2012). Comportamiento delictivo reincidente. Análisis diferencial de la variable edad. Revista Interamericana de Psicología, 46(3), 365-374.

Calafat, A., Fernández, C., Juan, M., \& Becoña, E. (2007). Weekend nightlife recreational habits: Prominent intrapersonal 'Risk factors' for drug use? Substance Use \& Misuse, 42, 1443-1454. DOI: http://dx.doi. org/10.1080/10826080701209044

Calvete, E., \& Estévez, A. (2009). Consumo de drogas en adolescentes: El papel del estrés, la impulsividad y los esquemas relacionados con la falta de límites. Adicciones, 21(1), 49-56. DOI: http://dx.doi.org/10.20882/ adicciones. 251

Cava, M. J., Murgui, S., \& Musitu, G. (2008). Diferencias en factores de protección del consumo de sustancias en la adolescencia temprana y media. Psicothema, 20(3), 389-395.

Cifuentes, J. J., \& Londoño, N. H. (2011). Perfil cognitivo y psicopatológico asociados a la conducta antisocial. International Journal of Psychological Research, 4, 58-69.

Contreras, L., Molina, V., \& Cano, M. C. (2011). In search of psychosocial variables linked to the recidivism in young offenders. The European Journal of Psychology Applied to Legal Context, 3, 77-88.

Contreras, L., Molina, V., \& Cano, M. C. (2012). Consumo de drogas en adolescentes con conductas infractoras: análisis de variables psicosociales implicadas. Adicciones, 24(1), 31-38.

Cortés, M. T., Espejo, B., Martín, B., \& Gómez-Íñiguez, C. (2010). Tipologías de consumidores de alcohol dentro de la práctica del botellón en tres ciudades españolas. Psicothema, 22(3), 363-368.

Delisi, M., Angton, A., Behnken, M. P., \& Kusow, A. M. (2015). Do Adolescent Drug Users Fare the Worst? Onset Type, Juvenile Delinquency, and Criminal Careers. International Journal of Offender Therapy and Comparative Criminology, 9(2), 180-95.

Dussaillant, F. (2010). Comportamientos riesgosos entre los jóvenes: El caso de la actividad sexual. Centro de Estudios Públicos, 118, 107-177. 
Estévez, E., Murgui, S., Moreno, D., \& Musitu, G. (2007). Estilos de comunicación familiar, actitud hacia la autoridad institucional y conducta violenta del adolescente en la escuela. Psicothema, 19(1), 108-113.

ESTUDES, (2014/2015). Encuesta Estatal sobre Uso de Drogas en Estudiantes de Enseñanzas Secundaria. Madrid: Ministerio de Sanidad y Política Social.

Faílde, J. M., Dapía, M. D., Alonso, A., \& Pazos, E. (2015). Consumo de drogas en adolescentes escolarizados infractores. Educación XX1, 18(2), 167-188. DOI: http:// dx.doi.org/10.5944/educXX1.14014

Farke, W., \& Anderson, P. (2007). Binge drinking in Europe. Adicciones, 19(4), 333-340.

Galicia, M., Alonso, J.R., \& Nogué, S. (2014). Intoxicaciones por drogas de abuso: substancias emergentes en el siglo XXI. Emergencias, 26, 472-480.

García, M. L., Tomás, S., González, L., Castillo, I., Mars, L., \& Balaguer, I. (2012). Estudio descriptivo longitudinal sobre el consumo de sustancias en la adolescencia. Revista Española de Drogodependencias, 1, 63-80.

Gázquez, J. J., Pérez-Fuentes, M. C., Molero, M. M., Barragán, A. B., Martos, A., \& Sánchez, C. (2016). Drug use in adolescents in relation to social support and reactive and proactive aggressive behavior. Psicothema, 28(3), 318322. DOI: http://dx.doi.org/10.7334/psicothema2015.327

Isorna, M., Fariña, F., Sierra, J. C., \& Vallejo-Medina, P. (2015). Binge drinking: conductas sexuales de riesgo y drogas facilitadoras del asalto sexual en jóvenes españoles. Summa Psicológica, 22(1), 1-8. DOI: http://dx.doi.org/10.1016/j. sumpsi.2015.05.001

Laespada, M. T. (2010). La dimensión sincrónica del deber en la España de hoy. Los menores como punto de especial atención y protección social ante el alcohol. En J. Elzo (Coord.), Hablemos de alcohol (pp. 13-34). Madrid: Entimema.

Loeber, R. (1988). Natural histories of conduct problems, delinquency and associated substance use: evidence for developmental progressions. En B.B. Lahey and A.E. Kazdin (Eds.), Advances in clinical child psychology (pp. 73-124). New York: Plenum.

Londoño, C., \& Valencia, C. (2012). Asertividad, resistencia a la presión de grupo y consumo de alcohol en universitarios. Acta Colombiana de Psicología, 15(1), 131-141.

López-Núñez, C., Fernández-Artamendi, S., FernándezHermida, J. R., Campillo, A., \& Secades-Villa, R. (2012). Spanish adaptation and validation of the Rutgers Alcohol Problem Index (RAPI). International Journal of Clinical and Health Psychology, 12(2), 251-264. DOI: http://dx.doi. org/10.1016/j.ijchp.2015.07.002

López, S., \& Rodríguez-Arias, J. L. (2010). Factores de riesgo $\mathrm{y}$ de protección en el consumo de drogas en adolescentes y diferencias según edad y sexo. Psicothema, 22(4), 568-573.
Luengo, M. A., Kulis, S., Marsiglia, F. F., Romero, E., GómezFraguela, J. A., Villar, P., \& Nieiri, T. (2008). A Cross-national study of preadolescent substance use: Exploring differences between youth in Spain and Arizona. Substance Use \& Misuse, 43, 1571-1593. DOI: http://dx.doi. org $/ 10.1080 / 10826080802241078$

Lyons, A., \& Willott, S. A. (2008). Alcohol consumption, gender identities and women's changing social positions. Sex Roles, 59, 694-712. DOI: http://dx.doi.org/10.1007/s11199008-9475-6

Martínez-Loredo, V., Fernández- Hermida, J. R., FernándezArtamendi, S., Carballo, J. L., \& García-Rodríguez, O. (2015). Spanish adaptation and validation of the Barratt Impulsiveness Scale for early adolescents (BIS-11-A). International Journal of Clinical and Health Psychology, 15, 274282. DOI: http://dx.doi.org/10.1016/j.ijchp.2015.07.002

Moral, M. V., \& Ovejero, A. (2009). Experimentación con sustancias psicoactivas en adolescentes españoles: perfil de consumo en función de los niveles de edad. Revista Latinoamericana de Psicología, 41(3), 533-554.

Moral, M. V., \& Ovejero, A. (2011). Consumo abusivo de alcohol en adolescentes españoles: tendencias emergentes y percepciones de riesgo. Universitas Psychologica, 10(1), 71-87.

Moral, M. V., Rodríguez, F. J., \& Sirvent, C. (2006). Factores relacionados con las actitudes juveniles hacia el consumo de alcohol y otras sustancias psicoactivas. Psicothema, 18(1), 52-58.

Moral, M. V., Bringas, Ovejero, A., Morales, L. A., \& Rodríguez, F. J. (2017). Emergencia sociosanitaria en consumo de riesgo de alcohol y síntomas de dependencia en jóvenes. Health and Addictions: Salud y Drogas, 17(2), 91-99. DOI: http://dx.doi.org/10.21134/haaj.v17i2.307

Morales, L. A., Bringas, C., Moral, M. V., Rodríguez, F. J., Cetz, C., \& Arriaga, L. O. (2015). Actitudes de permisividad y resistencia, motivaciones y percepciones de riesgo ante el consumo de alcohol en estudiantes mexicanos. Revista Española de Drogodependencias, 40(4), 12-29.

Motos, P., Cortés, M. T., Giménez, J. A., \& Cadaveira, F. (2015). Predictores del consumo semanal de alcohol y sus consecuencias asociadas en universitarios consumidores intensivos de alcohol. Adicciones, 27(2), 119-31. DOI: 10.20882/adicciones. 700

Mulvey, E. P., Schubert, C. A., \& Chassin, L. (2010). Substance use and delinquent behavior among serious adolescent offenders. Juvenile Justice Bulletin, 1-15. Recuperado de http://www.ncjrs.gov/pdffiles1/ojjdp/232790.pdf

Muñoz, J. M., Manzanero, A. L., Alcázar, M. A., González, J. L., Pérez, M. L., \& Yela, M. (2011). Psicología Jurídica en España: Delimitación Conceptual, Campos de Investigación e Intervención y Propuesta Formativa dentro de la Enseñanza Oficial. Anuario de Psicología Jurídica, 21, 3-14. 
Musitu, G., Suárez, C., Del Moral, G., Martínez, B., \& Villarreal, M. E. (2015). El consumo de alcohol en adolescentes: el rol de la comunicación, el funcionamiento familiar, la autoestima y el consumo en la familia y amigos. Revista Científica Divulgativa Búsqueda, 14, 45-61.

Natera, G., Juárez, F., Medina, M. E., \& Tiburcio M. (2007). Alcohol and drug consumption, depressive features, and family violence as associated with complaints to the Prosecutor's Office in Central Mexico. Substance Use and Misuse, 42, 1485-1504. DOI: http://dx.doi. org/10.1080/10826080701202817

Observatorio Español de la Droga y las Toxicomanías (2015a). Encuesta Domiciliaria sobre Alcohol y otras Drogas (EDADES) (2013/2014). España: Ministerio de Sanidad, Servicios Sociales e Igualdad. Recuperado de www.msssi. gob.es/gabinete/notasPrensa.do? $i d=3581$

Observatorio Español de la Droga y las Toxicomanías (2015b). Encuesta estatal sobre uso de drogas en Enseñanzas Secundarias. Madrid: Plan Nacional sobre Drogas.

Organización Mundial de la Salud (2014). Informe sobre la salud en el mundo. Estadísticas sanitarias mundiales. Recuperado el 17 de junio de 2016 de: www.who.int/gho/ publications/world_health_statistics/es/

Oquendo, M., Baca-García, E., Graver, R., Morales, M., Montalvan, V., \& Mann, J. (2001). Spanish adaptation of the Barratt Impulsiveness Scale (BIS-11). European Journal of Psychiatry, 15(3), 147-155. DOI: http://dx.doi. org/10.1016/j.ijchp.2015.07.002

Pedreira, J. L., Blanco, V., Pérez-Chacón, M. M., \& Quirós, S. (2014). Psicopatología en la adolescencia. Medicine, 11(61), 3612-3621. DOI: http://dx.doi.org/10.1016/S03045412(14)70821-2

Peters, E. N., Nordeck, C., Zanetti, G., O’Grady, K. E., Serpelloni, G., Rimondo, C., Blanco, C., Welsh, C., \& Schwartz, R. P. (2015). Relationship of gambling with tobacco, alcohol, and illicit drug use among adolescents in the USA: Review of the literature 2000-2014. American Journal on Addition, 24, 206-216. DOI: http://dx.doi. org/10.1111/ajad.12214

Pozo, R. (2012). La violencia de las mujeres jóvenes que delinquen: ¿violentas o violentadas? RES, Revista de Educación Social, 15, 1-12.

Ramírez, C. (2003). La transmisión intergeneracional, la clase del vínculo y los factores intrapersonales como predictores de la co-ocurrencia de comportamientos violentos y adictivos en jóvenes. Acta Colombiana de Psicología, 9, 51-69.

Rechea, C. (2008). Conductas antisociales y delictivas de los jóvenes en España. Castilla-La Mancha: Universidad de Castilla-La Mancha.
Ribas, M., del Prado, N., Claramunt, J., Civit, M., Canalias, O., \& Santaolalla,A., (2015). Adolescentes multiproblemáticos: consumo de tóxicos y trastorno mental en jóvenes que delinquen. Actas Españolas de Psiquiatría, 43(6), 197-204.

Rodríguez, F. J., Ovejero, A., Bringas, C., \& Moral, M. V. (2016). Afrontamiento de conflictos en la socialización adolescente. Propuesta de un modelo. Psicología desde el Caribe, 33(1), 1-13. DOI: http://dx.doi.org/10.14482/ psdc.33.1.8083

Rodríguez, F. J., Rodríguez-Franco, 1., López-Cepero, J., \& Bringas, C. (2012). Juvenile delinquency and Young ofender: bibliographical and bibliometric review of two perspectives of study. The European Journal of Psychology Applied to Legal Contex, 2(2), 117-143.

Rodríguez, F. J., Bringas, C., Moral, M. V., Pérez, B., \& Estrada, C. (2012). Consumo de sustancias psicoactivas y delito: análisis de la relación entre edad de inicio y reincidencia. International Journal of Psychological Research, 5(2), 58-65.

Romo, N., Marcos, J., Gil, E., Marquina, A., \& Tarragona, A. (2015). Bebiendo como chicos: consumo compartido de alcohol y rupturas de género en poblaciones adolescente. Revista Española de Drogodependencias, 40(1), 13-28.

Sanabria, A. M., \& Uribe, A. F. (2009). Conductas antisociales $\mathrm{y}$ delictivas en adolescentes infractores y no infractores. Pensamiento Psicológico, 6(13), 203-218.

Sánchez, A., Redondo, S., García, M. I., \& Velázquez, A. (2012). Episodios de urgencia hospitalaria relacionados con el consumo de alcohol en personas de entre 10 y 30 años en Castilla y León durante el período 2003-2010. Revista Española de Salud Pública, 86(4), 409-417.

San Juan, D., Ocáriz, E., \& Germán, I. (2009). Menores infractores y consumo de drogas: Perfil psicosocial y delictivo. Revista Criminalidad, 51, 147-162.

Scoppetta, O., Pérez, A., \& Lanziano, C. (2011). Perfiles asociados al consumo de alcohol en adolescentes escolarizados mediante análisis de correspondencias múltiples. Acta Colombiana de Psicología, 14(1), 139-146.

Velázquez, M., Arellanez, J. L., \& Martínez, A. L. (2012). Asertividad y consumo de drogas en estudiantes mexicanos. Acta Colombiana de Psicología, 15(1), 131-141.

Villarreal-González, M., Sánchez-Sosa, J. C., \& Musitu, G. (2013). Análisis psicosocial del consumo de alcohol en adolescentes mexicanos. Universitas Psychologica, 12(3), 15-31. DOI: http://dx.doi.org/10.11144/Javeriana.UPSY123.apca

White, H. R., \& Labouvie, E. W. (1989). Towards the assessment of adolescent problem drinking. Journal of Studies on Alcohol, 50, 30-37. DOI: http://dx.doi.org/10.15288/ jsa.1989.50.30 